\title{
Information et économie publique
}

\section{Jean-Jacques Laffont}

\begin{abstract}
Information and public economics by Jean- Jacques Laffont

Taking into account operators' strategic behaviour in response to their private information and the inefficiencies in public decision-making due to the operations of interest groups leads to a more realistic vision of State intervention. Taking four examples - the regulation of natural monopolies, public purchasing policies, the production of public goods and the economics of the environment, the author illustrates the main stages of this new methodology in the field of public economics.
\end{abstract}

\section{Résumé}

Information et économie publique par Jean- Jacques Laffont

La prise en compte du comportement stratégique des acteurs vis-à-vis de leur information privée et des inefficacités de la décision publique dues au jeu des groupes d'intérêt a conduit à une vision plus réaliste de l'intervention de l'État. Sur quatre exemples, la régulation des monopoles naturels, les politiques publiques d'achat, la production de biens publics et l'économie de l'environnement, l'auteur illustre les grandes étapes de cette méthodologie nouvelle de l'économie publique.

\section{Citer ce document / Cite this document :}

Laffont Jean-Jacques. Information et économie publique. In: Économie \& prévision, n¹45, 2000-4. pp. 107-115;

doi : $10.3406 /$ ecop.2000.6117

http://www.persee.fr/doc/ecop_0249-4744_2000_num_145_4_6117

Document généré le 17/06/2016 


\section{Information et économie publique}

Jean-Jacques Laffont ${ }^{(*)}$
J'ai tenté de rassembler ici quelques idées sur la façon dont l'économie publique a été amenée à introduire les considérations de l'information au cours des dernières années. Jusqu'à une date récente, on peut dire que l'économie publique et de manière générale la politique économique ont été conçues dans le cadre du paradigme de l'État informé et bienveillant. Cette approche a produit de nombreux enseignements, qu'il ne faut pas regretter, comme, par exemple, le rôle des taxes pigouviennes pour internaliser les externalités, la nécessité de satisfaire les conditions de Samuelson pour optimiser l'allocation des biens publics, l'insuffisance des taxes linéaires pour contrôler les entreprises à technologie non convexe, comme les monopoles naturels, pour n'en citer que quelques-uns.

Mais cette approche a aussi conduit à une vision trop optimiste de la politique économique. En relâchant les hypothèses de bienveillance et d'information parfaite de l'État, l'économie publique contemporainc a ćtć complc̀tement renouvelée et débouche, aujourd'hui, sur une vision plus réaliste de l'intervention économique de l'État.

La première étape consiste à prendre en compte les barrières informationnelles qui contraignent l'intervention de ce dernier. L'approche normative traditionnelle a été étendue, par la littérature de la théorie des mécanismes, à un monde où l'État bienveillant est contraint par des conditions incitatives concernant le comportement des agents économiques. De nombreux enseignements ont été tirés de cette analyse sur la nature des distorsions souhaitables de l'allocation des ressources pour traiter au mieux ces contraintes informationnelles.

De manière générale, il est apparu que l'information privée des agents économiques est pour eux une source de rentes dites "rentes informationnelles", qui sont coûteuses pour l'État ou le décideur collectif concerné. Celui-ci doit donc concevoir des règles du jeu qui arbitrent de façon optimale entre la distribution coûteuse de ces rentes et les inefficacités également coûteuses qui les atténuent. Une analyse systématique des questions de politique économique de ce point de vue normatif, qui prend donc en compte la décentralisation de l'information et le comportement stratégique des agents économiques, est en cours. Mais elle n'est pas achevée, même dans les cadres les plus simples ainsi que dans des domaines comme l'économie de la santé, l'économie de l'éducation, l'économie de l'environnement et l'économie sociale en général.

Une première étape de ce travail doit se dérouler dans le cadre assuré de la théorie des contrats complets qui présume, entre autres, l'absence de coûts liés à la complexité des contrats, l'absence de difficultés à s'engager dans l'avenir, l'absence de comportement collusif, la disponibilité de juges bienveillants, l'absence de coûts de communication ou de
La retranscription de cette conférence a été effectuée par $\mathrm{M}$. Le Goff et T. Giguelay du groupe de recherche ICI de l'UBO. $n^{\circ} 1452000-4$ 
rationalité limitée. Dans une deuxième étape, il faut relâcher pas à pas ces hypothèses irréalistes pour entrer dans le monde moins bien assuré, du point de vue méthodologique, des contrats incomplets.

L'approche de la théorie des mécanismes par les contrats complets est l'analogue de la théorie des marchés complets d'Arrow-Debreu. Elle constitue un cadre de référence incontournable aujourd'hui. Prendre en compte la non-bienveillance de l'État, c'est-à-dire sa capture par les groupes d'intérêt, nous conduit ensuite à l'économie politique de l'économie publique. Du point de vue méthodologique, cette étape supplémentaire peut être vue comme un cas particulier de la théorie des contrats incomplets. En effet, une constitution choisie ex ante par les citoyens derrière le voile de l'ignorance pourrait constituer cet état bienveillant dont j'ai parlé précédemment. Mais de par sa simplicité (qu'il conviendrait d'expliquer), la constitution n'est pas ce contrat complet qui permet de contrôler les politiciens. Au contraire, elle leur laisse beaucoup de discrétion, ce qui leur permet de favoriser certains groupes d'intérêt. En d'autres termes, la nécessité de décentraliser la politique économique à des hommes politiques crée un nouveau problème incitatif qui doit conduire, au niveau constitutionnel, à des règles d'économie publique nouvelles.

Dans mon exposé, je vais tenter d'illustrer cette méthodologie en considérant, certes très rapidement, les quatre domaines de l'économie publique que sont la réglementation des monopoles naturels, les politiques publiques d'achat, la production de biens publics et enfin l'économie de l'environnement. Je commencerai en illustrant l'approche "mécanismes » de la politique économique par la théorie de la réglementation des monopoles naturels.

Avec le travail de Loeb et Magat de 1979 ou de Baron et Myerson de 1982, est apparue clairement la difficulté essentielle de la réglementation des monopoles naturels qui provient de l'information asymétrique existant entre le régulateur et le monopole, en particulier en ce qui concerne la fonction de coût. Le problème a été formalisé sous forme de modèle principal-agent, dans lequel le principal est le régulateur et l'agent l'entreprise. Dans le cadre de la théorie des contrats complets, on sait alors, d'après le principe de révélation, que toute forme de réglementation est équivalente à un mécanisme de révélation incitatif.

Ce mécanisme de révélation est conçu pour extraire de façon incitative l'information privée de l'entreprise en s'engageant sur une utilisation bien définie de cette information. Dans ce cas particulier, on s'engage sur une certaine quantité de biens à produire par l'entreprise et sur un certain transfert monétaire entre le régulateur et l'entreprise, ceci en fonction de l'information transmise. La première étape de l'analyse consiste à caractériser l'ensemble des mécanismes de révélation incitatifs, ou encore, l'ensemble des contraintes incitatives découlant de l'existence d'informations privées, qui sont des contraintes supplémentaires qu'imposent les asymétries d'information sur l'allocation des ressources en raison de la décentralisation de l'information. On définit ainsi l'ensemble des allocations réalisables et incitatives de l'économie qui caractérisent ce qui est vraiment réalisable quand on prend en compte les incitations des acteurs. Et j'insiste sur le fait que, grâce au principe de révélation et à cette démarche, on peut caractériser l'ensemble des réalisables incitatifs. Ce sont des conditions nécessaires et suffisantes.

Dans le modèle particulier de Baron et Myerson, ces contraintes spécifient d'une part que le niveau de production doit être une fonction décroissante du coût marginal (ce qui correspond aux conditions incitatives du deuxième ordre dans le jargon de la théorie des incitations) et d'autre part que le transfert du régulateur à l'entreprise est déterminé à une constante près par le profil croissant de production que l'on désire mettre en œuvre.

La seconde étape de l'analyse normative, une fois que l'on a caractérisé l'ensemble des allocations incitatives possibles, consiste alors à maximiser le bien-être social espéré dans cet ensemble pour déterminer le mécanisme de révélation incitatf optimal. Baron et Myerson, par exemple, montrent de façon précise comment le niveau de production doit être diminué par rapport au niveau optimal en information complète pour décroître la rente informationnelle capturée par l'entreprise. En fait, la tarification au coût marginal n'est plus optimale : le prix doit être égal à un coût marginal virtuel qui revient, d'une certaine manière, à inclure dans les coûts celui de la rente informationnelle que l'on est obligé d'abandonner à l'entreprise.

Dans Laffont-Tirole (1986), ce modèle a été généralisé en supposant une double asymétrie d'information : un paramètre de coût qui, comme dans celui de Baron et Myerson, représente une information cachée et une variable de risque moral (l'effort de l'entreprise qui lui permet de diminuer le coût). Dans la version la plus simple de ce modèle qui suppose l'observabilité ex post des coûts, le coût est égal à ce paramètre de sélection adverse moins le niveau d'effort. Une fois ce coût observé, on est ramené techniquement à un modèle d'information cachée ou de sélection adverse. Mais ce modèle est beaucoup plus adapté à l'analyse de la réglementation des monopoles car, dans la réglementation habituelle, on fait une forte utilisation de l'obscrvation des coûts. Jusqu'au price-cap récent, l'essentiel des procédures de régulation était basé sur le remboursement des coûts, de telle sorte qu'il n'était pas possible de modéliser 
ce problème avec le modèle de Baron et Myerson (qui suppose justement que les coûts ne sont pas observables).

Une des conclusions importantes de l'analyse de ce modèle est que le mécanisme optimal de réglementation prend la forme de contrats intermédiaires entre des contrats à prix fixes (qui favorisent l'effort) et des contrats de partage de coût (qui peuvent aller jusqu'aux contrats cost-plus), contrats qui ont l'avantage de limiter fortement les rentes que l'on abandonne, avec, bien sûr, l'inconvénient de ne pas induire un bon niveau d'effort. Voilà les enseignements qu'on peut retirer de ces analyses en information asymétrique. On s'aperçoit aussi qu'à la différence du modèle de Baron-Myerson dans lequel la tarification doit dévier des règles habituelles du coût marginal, ceci n'est plus toujours nécessaire ici car le problème des incitations est réglé par le remboursement des coûts.

Il scrait important d'ćtendre cette analyse, développée dans un premier temps pour des entreprises neutres au risque, au cas d'entreprises avec de l'aversion au risque. Ceci est particulièrement important si l'on veut travailler sur des problèmes d'assurance ainsi que dans de nombreux problèmes d'économie publique comme la santé dans lequel ces considérations d'assurance sont essentielles. Nous avons amorcé (dans Laffont-Rochet, 1998) cette analyse en généralisant la démarche ci-dessus au cas d'entreprises qui ont de l'aversion au risque, mais malheureusement en se limitant au cas où l'observation des coûts évoquée précédemment n'est pas bruitée. Dans le cas général, qui nous amènerait donc à nous intéresser à des problèmes de contrôle public dans lesquels on a simultanément des problèmes de sélection adverse, de risque moral et de partage de risque (pour des agents qui ont de l'aversion au risque), on ne dispose pas à l'heure actuelle d'une théorie générale très satisfaisante et il faut, comme le font McAfee et McMillan (1987), s'en tenir à des mécanismes linéaires pour dériver quelques intuitions sur ce que peut être une bonne réglementation. Il y a donc là encore beaucoup à faire.

Bien que l'on puisse élargir la réglementation à l'aide d'enchères ex ante (comme dans le cas de la télévision par câble, par exemple), ainsi que le préconisait déjà Walras (idée que les Américains attribuent à Demsetz), cela reste relativement rare et c'est dans le domaine des offres publiques d'achat que les enchères sont le plus souvent utilisées.

Dans ce cas, le modèle doit être élargi à une situation entre un principal et plusieurs agents. Le principal va alors tenter d'utiliser la concurrence entre agents pour limiter les rentes informationnelles qu'il doit abandonner et, jusqu'à présent, on a supposé en économie publique que les agents, qui sont eux-mêmes en information incomplète les uns par rapport aux autres, ont un comportement non coopératif de Nash ou de Nash Bayésien.

C'est ainsi que Myerson en 1981 a caractérisé les enchères optimales pour un vendeur d'un bien indivisible dans le modèle à valeurs indépendantes de Vickrey en utilisant la théorie des mécanismes. Comment organiser des politiques publiques d'achat dans le cas simple d'une enchère d'un bien indivisible. Dès que les caractéristiques des agents sont corrélées, le principal peut construire des mécanismes qui extraient tout le surplus. Tel est le sens du théorème de Crémer-McLean de 1988 qui aboutit à ce résultat quelque peu extrême selon lequel, dès que les agents se connaissent un peu, ou qu'ils ont des informations liées les unes aux autres, le principal peut, en construisant des mécanismes suffisamment astucieux, éliminer toutes les rentes informationnelles qu'il était obligé d'abandonner lorsqu'il n'y avait qu'un seul agent. Bien sûr, ce résultat reste très fortement basé sur la neutralité au risque, sur l'absence de contrainte de responsabilité limitée ainsi que sur l'absence de comportement collusif. En utilisant des généralisations assez simples de Myerson, nous avons étendu avec Tirole (1987) la réglementation optimale au cas des enchères de contrats (cas le plus adapté pour les offres publiques d'achat).

Si l'on évoque maintenant la théorie des biens publics, que s'est il passé ? L'analyse du passager clandestin et de la production des biens publics a été en fait un domaine pionnier de la théorie des mécanismes bien avant la théorie de la régulation. Mais ce cas-là correspondait déjà à un contexte assez compliqué puisque c'est une situation avec un principal et plusieurs agents au-delà de celui de la régulation de type Baron et Myerson entre un principal et un agent : l'État fait face à de multiples agents désireux de consommer le bien public . La première vague de travaux de l'époque, issue de Groves (1973), a eu deux faiblesses : la première a été de se restreindre aux mécanismes à stratégie dominante qui sont plus faciles à manier, et la deuxième de se concentrer sur la réalisation de la décision de biens publics optimale en information parfaite. Donc, malgré l'information asymétrique, on espérait faire des choix collectifs comme s'il n'y avait pas de coûts supplémentaires.

Dans mon livre avec Green (1979), nous avions déjà montré que ces mécanismes de Groves n'étaient pas équilibrés, que l'on n'était donc pas vraiment en l'équilibre général, ce qui posait problème. Il fallait donc s'écarter de la quantité optimale de biens publics en information parfaite puisque, de toutes façons, on était en second rang, car, le budget n'était pas équilibré. Aspremont et Gérard-Varet ont apporté en 1979 une contribution importante en adoptant le cadre Bayésien, ce qui permet de passer de l'implémentation (ou mise en œuvre) en stratégie dominante à l'implémentation en équilibre 
Bayésien. Ils ont alors montré que ce problème d'équilibre budgétaire n'en était plus un si on acceptait d'affaiblir la force des incitations requises. L'étape suivante a été de remarquer, comme je l'avais fait avec Maskin en 1979, que même si on utilise les mécanismes Bayésiens qui nous permettent d'assurer l'équilibre budgétaire, si on veut continuer à mettre en œuvre la décision publique optimale en information parfaite, on ne peut pas satisfaire les conditions de rationalité individuelle, autrement dit on ne peut pas avoir l'accord des participants au mécanisme. Rob (1989), Mailath et Postlewaite (1990) ont ensuite montré une forme extrême de ce résultat en faisant croître le nombre d'agents et en s'apercevant qu'on arrivait à très peu de choses. Quand on impose que les agents acceptent de participer, et que l'équilibre budgétaire soit réalisé, on retrouve la forme moderne du problème du passager clandestin à savoir que l'on ne peut pas, et de loin, réaliser la décision publique optimale, même avec les mécanismes les plus sophistiqués du monde que nous apportent lc principe de révélation, et qu'il y a donc bien un problème sérieux avec les biens publics y compris quand on n'a pas de contraintes sur les mécanismes que l'on peut utiliser.

En économie de l'environnement, on s'est très vite aperçu que le problème était très proche de celui des biens publics. Je renvoie sur ce thème à Dasgupta, Hammond et Maskin (1980), le sujet ayant très peu évolué jusqu'à un article de Baron de 1985, puis une littérature très récente et un peu plus systématique sur l'utilisation de la théorie de mécanismes dans les problèmes d'environnement. À titre d'exemple, on peut citer un article (Laffont et Tirole (1996)) sur l'approche mécanismes des droits de pollution, la question posée étant : dans quelle mesure la création de marchés de droits de pollution va-t-elle conduire les agents à ne pas suffisamment investir dans les techniques de dépollution ? C'est le genre de problèmes que l'on peut aborder avec la théorie des mécanismes.

Voici donc quelques exemples de la première phase de la démarche. Face à un problème d'information asymétrique, l'État, dont on continue de supposer qu'il est bienveillant, cherche à maximiser le bien-être social et construit un mécanisme, certes un peu compliqué et abstrait, sous forme d'un mécanisme de révélation incitatif. On connaît parfaitement la démarche à suivre : il suffit alors d'écrire les conditions incitatives et d'optimiser dans cet ensemble d'allocation réalisables et incitatives. Une fois que l'on a ainsi obtenu le mécanisme de révélation incitatif optimal, se pose la question de sa mise en œuvre, de son implémentation. Au delà de ce mécanisme de révélation abstrait dans lcquel chacun annonce à l'État ses préférences et ce dernier envoie en retour des ordres, on est conduit à s'interroger sur l'existence d'institutions qui pourraient réaliser la même chose que ce mécanisme, ou qui, si elles ne le réalisent pas, pourraient être comparées à cette institution optimale dérivée de la théorie. Prenons des exemples afin d'illustrer notre propos.

Dans le cas du problème de régulation de Baron et Myerson, le mécanisme de révélation optimal, qui pourrait paraître comme une construction de théoriciens fous, est en fait équivalent à un transfert non linéaire, c'est-à-dire à un paiement fonction non linéaire de la quantité produite. C'est totalement équivalent. Ce prix non-linéaire va être concave ou convexe selon les cas, mais il y a une identité entre l'objet, mécanisme de révélation incitatif, et l'objet, prix non-linéaire. On a en quelque sorte ici la représentation institutionnelle parfaite du mécanisme de révélation. Dans mon article avec Tirole de 1986, le mécanisme de révélation optimal peut être implémenté par un menu de contrats linéaires, donc un menu de partage de coûts. Ce n'est donc pas un objet abstrait mais tout simplement l'offre par le principal d'un monu de contrats : il peut ainsi proposer le choix entre une option dans laquelle les coûts sont remboursés à $50 \%$ mais avec un paiement fixe de $100 \mathrm{~F}$ et une deuxième option avec un remboursement à $10 \%$ et un paiement fixe de 200F. Ainsi, l'implémentation du mécanisme optimal dérivé de la théorie générale se fait sous une forme institutionnelle assez convenable, qui est souvent un menu de contrats linéaires. Dans la pratique, pour poursuivre un peu ce point, seul un petit nombre d'options en général est offert ; on peut tenter d'en évaluer la performance. Évaluer la performance consiste par exemple à se demander si l'on perd beaucoup en n'offrant que trois contrats par rapport à un continu de contrats ? Dans un contexte un peu différent, Wilson (1993) a ainsi étudié comment la perte varie avec le nombre d'options dans le cas du tarif d'un monopole quand on a une population hétérogène de consommateurs ? On s'aperçoit qu'on n'a pas besoin d'un grand nombre d'options pour arriver à extraire à peu près l'ensemble du surplus. Dans un article de 1994, Gasmi, Ivaldi et Laffont ont fait de la même manière une analyse par simulation qui permet de comparer des réglementations plus ou moins réalistes par rapport à la réglementation optimale dérivée de la théorie. C'est ainsi qu'on peut savoir si en utilisant un price cap on perd $10 \%$ ou $5 \%$ de bien-être social par rapport à ce qu'on aurait, si on avait réussi à mettre en place le mécanisme optimal.

Dans le cas des enchères, comment se passe ce problème d'implémentation ? Il s'avère (ce qui relève presque du miracle) que dans de nombreux cas (il faut des hypothèses sur les distributions des dispositions à payer), les enchères traditionnelles au premier prix ou enchères orales ascendantes, descendantes au premier ou second prix, implémentent toutes le mécanisme optimal. Il se trouve que ces institutions sont optimales, au moins tant que les acheteurs sont neutres au risque. Par 
contre, dès que les acheteurs se mettent à avoir de l'aversion au risque, on s'aperçoit que l'enchère au premier prix devient supérieure à l'enchère au second prix ou enchère de Vickrey, et donc il y a divergence entre ces enchères mais surtout qu'aucune d'elles n'est alors optimale. On pourrait prendre des exemples pour savoir combien on perd par rapport à ce que serait une enchère optimale. De la même façon, la détermination pratique des niveaux de biens publics s'effectue la plupart du temps par des procédures de vote et non par des mécanismes de Groves, c'est-à-dire des mécanismes sans transfert. Or, on sait qu'avec des préférences unimodales, le vote majoritaire a d'excellentes propriétés incitatives puisque annoncer ses vraies préférences est une stratégie optimale avec une bonne efficacité si la moyenne des valuations n'est pas trop loin de la médiane.

Moulin a montré en 1980 que, dans un contexte de mécanismes sans transfert, les seuls mécanismes non manipulables sont des dictateurs positionnels, le choix de l'agent médian en étant un exemple. Récemment, pour revenir à des applications, certains inputs publics comme le spectre hertzien ont été alloués par des mécanismes complexes d'enchères avec transfert aux États-Unis, ce qui a constitué un immense succès financier tant pour l'État que pour les économistes. Beaucoup pensent que les économistes ont acquis plus de prestige avec cette affaire qu'avec tout ce qu'ils avaient pu faire avant et cela montre que la micro-économie peut servir à quelque chose.

Dans le cas de l'environnement, puisque je veux faire le tour de ces implémentations, j'ai montré dans un exemple (Laffont, 1994) que le mécanisme optimal de révélation, qui est quelque chose d'assez abstrait dans le cas d'un monopole pollueur, peut être mis en œuvre par la combinaison d'une taxe linéaire à la production, d'une taxe non linéaire sur le niveau de pollution et d'un transfert monétaire, tous trois fonction du coût observé. On peut trouver là aussi des institutions qui réalisent la mise en œuvre du mécanisme de révélation optimal. Ainsi, une fois que l'on sait à quoi ressemble le mécanisme optimal de révélation, on s'interroge dans une deuxième étape sur la possibilité de le mettre en œuvre et sur ce que l'on perd si on n'y parvient pas. C'est ce que j'appelle la démarche des contrats complets, l'approche "mécanismes de contrats complets". J'ai souligné que, dans cette approche, on a en général supposé que les agents, dans ce contexte principal multi-agents, ont des comportements non coopératifs, ce que l'on a modélisé par des équilibres de Nash Bayésiens.

Ce comportement de Nash Bayésien peut être justifié si les coûts de communication entre les agents sont très grands. Mais cela suppose en quelque sorte que le Principal, cet État bienveillant, ait aussi au fond le contrôle de la communication. Ce modèle serait adéquat si on était dans un monde où le principal peut vraiment couper les communications entre les agents. Cela semble un peu excessif et on souhaite donc en économie publique analyser les possibilités de comportements collusifs. Il y a actuellement deux approches de la collusion qui sont des instruments que l'on peut utiliser et qui peuvent faire encore l'objet de beaucoup d'applications.

Les deux approches de la collusion que je vais évoquer supposent que les parties qui font collusion peuvent signer des contrats. Cela constitue bien entendu un raccourci puisque ces contrats sont illicites et il n'y aurait pas de juges bienveillants pour les mettre en œuvre. Il faut en fait considérer ceci comme une étape. Comment donner des fondations plus solides à ces analyses ? Bien évidemment, par les jeux répétés; il est clair que ces collusions que l'on trouve dans les institutions, dans les organisations, ne sont pas soutenues par des contrats explicites mais le seront par des phénomènes de réputation. La démarche statique plus simple consiste à supposer, éventuellement avec des coûts de transaction, qu'on peut signer des contrats. Cette approche a été développée par Tirole en 1986 dans un modèle hiérarchique extrêmement important pour le cas de l'information vérifiable. Autrement dit, ce sont des situations avec un principal, un superviseur et un agent. Il y a un problème d'asymétrie d'information entre le principal et l'agent, et le principal embauche un superviseur pour essayer de limiter son asymétrie d'information. Par exemple, il va s'informer sur le coût de l'entreprise et ainsi diminuer l'asymétrie d'information. Néanmoins, ce superviseur, dans la modélisation de Tirole, s'il observe une information, va découvrir des preuves de cette information. On dit que l'information est hard, c'est à dire vérifiable.

Supposons qu'il y ait deux valeurs possibles de cette information $\beta$ et $\beta$. Il va peut être découvrir que c'est $\beta$ qui prévaūt et il aura alors des preuves pour le démontrer. Il ne pourra pas prétendre qu'il a observé l'autre valeur. Mais, ce qu'il pourra faire, c'est de dire qu'il n'a rien observé puisque, dans la modélisation, on peut ne rien observer avec une certaine probabilité. Ainsi, la discrétion du superviseur, c'est de laisser son rapport "dans le tiroir". Cette approche astucieuse a une grande vertu qui est de transformer des problèmes de sélection adverse, dans lesquels on pourrait mentir dans toutes les directions, en un simple problème de risque moral avec essentiellement une seule équation qui consiste à savoir si oui ou non il faut cacher le rapport dans le tiroir. Dans ce contexte, Tirole peut démontrer un principe de robustesse à la collusion qui est une sorte de généralisation des principes de révélation. Autrement dit, s'il y a dans l'organisation une possibilité de collusion, on démontre alors que la meilleure chose que puisse faire l'État (le principal), c'est de construire un mécanisme tel que, non seulement les agents n'aient pas envie de mentir (ce 
qui représente les incitations individuelles traditionnelles), mais aussi tel qu'ils n'aient pas non plus intérêt à la collusion.

Ainsi, grâce à ce principe de robustesse à la collusion, on peut étendre la méthodologie constructive du principe de révélation puisqu'on peut reprendre la démarche en deux temps, c'est-à-dire écrire d'abord toutes les contraintes d'incitation individuelles et de collusion (qui assurent qu'il n'y aura pas collusion et que personne n'osera mentir), et on peut maximiser ensuite dans cet ensemble, ce qui est essentiel d'un point de vue méthodologique. En conséquence, ce modèle, qui est un cas très particulier, est néanmoins extrêmement puissant. Dans mon livre avec Tirole (1993) sur la théorie de la régulation, il en a été fait une grande utilisation, que ce soit pour étudier la corruption dans les appels d'offre, pour utiliser la manipulation des coûts comptables ou le favoritisme dans les enchères, etc. C'est donc un modèle extrêmement utile et c'était, jusqu'à récemment, le seul qui permettait, grâce à la caractérisation des allocations robustes à la collusion, d'aller jusqu'au calcul de la réponse régulatoire optimale face à une possibilité de collusion.

Ceci n'est toutefois valable que lorsque les agents envoient des messages de type hard et non de type soft (qui ne sont pas vérifiables). Dans un travail entrepris depuis deux ou trois ans (Laffont-Martimort (1998) par exemple), nous avons récemment construit une démarche qui a la même vertu méthodologique mais avec une information soft. Il s'agit d'un modèle principal deux agents dans lequel ces derniers peuvent faire de la collusion, cette fois sur la base d'informations non vérifiables. Chacun, par exemple, a des préférences et il y a échange d'information sur ces préférences, personne ne pouvant toutefois prouver quelles sont les préférences. On peut néanmoins démontrer un principe de robustesse à la collusion, c'est-à-dire tel que, pour le principal, la meilleure chose à faire est de construire des mécanismes qui ne conduiront pas à la collusion. C'est quand même le monde sympathique, où ce qui est optimal c'est qu'il n'y ait pas de corruption et ceci est très lié au fait que l'on est toujours dans un monde de contrats complets.

Ainsi, dans ce monde de contrats complets, il y a des méthodologies qui permettent de caractériser les réponses régulatoires face à des possibilités de collusion. Ceci permet de dépasser la naïveté de l'approche «mécanismes » précédente, qui suppose des comportements non coopératifs et postule que l'État peut, s'il le désire, faire en sorte que les agents se battent en duel pour profiter de leur confrontation. Or, ceux-ci comprendront assez vite qu'ils ont intérêt à s'allier contre lui. En introduisant explicitement la possibilité de collusion, on peut avancer dans la réflexion en économie publique.
Évoquons maintenant l'étape suivante. Une application majeure de l'approche de contrats complets est la supériorité d'une organisation centralisée de l'économie dans laquelle toute l'information est transmise par des mécanismes incitatifs à un centre qui envoie en retour des commandes ou des recommandations. En quelque sorte c'est la généralisation de la théorie bien connue de l'information complète qui montre l'équivalence équilibre-optimum. 'T'out ce qu'on peut faire avec le marché, on peut le faire de manière centralisée. C'est un peu plus fort comme résultat : même quand l'information est décentralisée et que les agents qui ont cette information ont des comportements stratégiques, s'il n'y a pas de restrictions sur les contrats (donc si on est bien dans le monde des contrats complets), on retrouve le fait que tout ce qu'on peut faire avec le marché, un État bienveillant qui centralise l' information peut le faire aussi. Or, à l'évidence, ce résultat entre en conflit avec le bon sens, ce que l'on peut dire aujourd' hui d'expérience. Ceci n'est pas étonnant, car il y a de nombrcuscs hypothèses explicites ou implicites qui sous-tendent le principe de révélation. J'ai évoqué précédemment cette longue liste de conditions mais revenons-y plus longuement.

La première, c'est évidemment l'hypothèse de rationalité illimitée des agents économiques et de coûts nuls de la communication qui ne met aucune limite à la complexité des contrats.

La deuxième, qui n'est pas suffisamment mise en avant à mon avis, est qu'on dispose de juges rationnels et bienveillants pour rendre les contrats exécutoires. Cela est quelque peu mythique.

Quel est alors le problème à ce stade ? Il faut évidemment aller vers les contrats incomplets, bien que nous n'ayons aucune méthode fondamentale pour relâcher ces hypothèses. Si on veut tenir compte de la rationalité limitée, il y a alors malheureusement une infinité de modèles de rationalité limitée dont aucun ne s'impose clairement, quand bien même c'est une facette importante de la réalité. Que recommander? Prendre des raccourcis, en adoptant des implications de l'absence de validité de ces hypothèses. Comme on ne sait pas modéliser la rationalité limitée, ou les coûts de communication, ou encore le fait que les juges ne sont pas nécessairement bienveillants ou rationnels, on peut prendre en compte des limites aux contrats qui sont, en fait, des implications de ces phénomènes fondamentaux.

On peut, par exemple, supposer que l'État ne peut pas s'engager au-delà d'un an (ou de quatre ans), alors que, dans la théorie précédente, il n'y avait aucune limite sur le pouvoir d'engagement de ces contrats. Cette incapacité à s'engager peut être liée à des problèmes de rationalité limitée, de coûts de communication, etc. Si l'on fait cette hypothèse 
qu'on ne peut s'engager que sur un an, et que l'on en déroule les implications pour le contrat optimal, on obtient une façon pragmatique d'approcher ce monde de contrats incomplets.

Une autre hypothèse, qui a remporté du succès dans la théorie des contrats, abordée la première fois par Dewatripont (1989) dans les modèles avec information cachée, réside dans le fait que l'on peut s'engager sans pour autant garantir que l'on ne renégocie pas par la suite pour améliorer le contrat. Ainsi, s'il suffit de passer devant un notaire pour signer un contrat entre les parties, aucune institution ne sera présente si l'année prochaine les deux parties décident de déchirer le contrat. On ne peut les en empêcher facilement, quand bien même cela serait utile pour pouvoir donner des incitations ex ante. De la même façon que précédemment, on peut caractériser le contrat optimal robuste à la renégociation, ce qui est un exercice en théorie des contrats incomplets non fondamentaliste. En effet, pour ce faire, il a fallu prendre un raccourci face à ce que l'on ne sait pas modéliser, qui consiste à supposer que les agents ne peuvent pas s'engager à ne pas renégocier. Ceci a été fait dans un certain nombre de domaines. Sans entrer dans les détails, il peut être utile à ce stade de prendre deux autres exemples d'approche indirecte de type contrats incomplets. Il s'agit d'une part de la théorie des multi-principaux : on peut prendre par exemple le Ministère de l'Industrie et celui de l'Environnement, chacun optimisant indépendamment, ce qui permet d'analyser les inefficacités du comportement non coopératif de ces deux ministères. Pourquoi s'agit-il de contrats incomplets? Parce qu'au fond il manque en amont un agent central qui harmoniserait les comportements de ces deux ministères. Il s'agit d'autre part de la non-vérifiabilité, approche la plus communément associée aux contrats incomplets. C'est l'approche retenue par des auteurs comme Grossman et Hart (1986), Aghion et Bolton (1992) etc., qui est un peu différente mais qui participe de la même démarche générale. Elle consiste à reconnaître qu'il y a ex post une connaissance commune sur la variable, mais qu'il n'y a pas de tierce partie pour la vérifier. Dans une démarche fondamentaliste de contrats complets, on construirait alors des mécanismes complexes à la Maskin qui permettraient d'éliminer les inconvénients de cette asymétrie d'information, mais qui supposent l'existence de juges bienveillants. Face à cette approche, Grossman-Hart supposent simplement que les contrats ne peuvent être conditionnés par cette variable. C'est évidemment une cote mal taillée puisqu'on pourrait définir ces mécanismes de révélation expost mais, là encore, c'est un raccourci d'une théorie des contrats complets. Je suis plus critique à l'égard de ce type de raccourci que par rapport à d'autres, non pas tant pour des raisons de principe que d'expérience. En effet, une fois que l'on s'octroie la liberté de décider à tout moment qu'une variable est vérifiable ou non-vérifiable on peut à peu près démontrer n'importe quoi, ce qui est inquiétant. Cette approche doit être maniée avec prudence au voisinage de modèles de contrats complets.

Je veux en venir maintenant pour conclure à l'absence de bienveillance de l'État. On devrait, de fait, consacrer une place plus importante à l'économie politique de l'économie publique. J'ai évoqué précédemment les illusions passées sur le fait que l'Etat soit parfaitement informé et qu'il maximise le bien-être social. Comment étudier cette seconde déviance par rapport à l'analyse traditionnelle ? On peut analyser cette économie politique comme un exercice en contrat incomplet. En effet, une manière de relâcher l'hypothèse d'un État bienveillant est de postuler que l'on ne néglige plus les problèmes incitatifs existant dans la délégation de la politique économique aux hommes politiques. L'incomplétude vient du caractère peu contraignant des contraintes constitutionnelles, lui-même dû à la simplicité contractuelle de la constitution.

Si l'on avait des juges bienveillants dans lesquels on avait totale confiance, on pourrait parfaitement contrôler les politiciens. Ainsi, pour des raisons fondamentales qu' on ne peut pas trop expliquer, il se trouve qu'on a une constitution extrêmement peu contraignante pour les politiques et c'est donc un contrat incomplet. On peut alors faire de l'économie politique en empruntant cette voie et en recherchant comment, en modifiant les institutions, on peut arbitrer entre cette discrétion des politiques et les moyens importants que l'on souhaite leur confier pour qu'ils soient efficaces. Cette insuffisance de l'analyse traditionnelle de l'économie publique avait déjà été soulignée par la Virginia Polytechnic School, par l'École de Chicago, et c'est pour cette contribution que Buchanan a reçu le prix Nobel. De fait, ceci a été souligné au tout début de l'économie de la régulation, bien avant que l'économie publique traditionnelle ne s'y intéresse. On a assisté à un vaste effort pour réintroduire les contraintes politiques dans l'analyse économique, en commençant par la macroéconomie pour s'étendre à toutes les disciplines, y compris en économie publique.

Je citerai deux exemples : on ne peut guère construire de théorie de la privatisation si on ne veut pas s'écarter du paradigme de l'État bienveillant. Shapiro et Willig (1990) ont ainsi développé un petit modèle dans lequel la privatisation des entreprises publiques crée une asymétrie d'information entre le pouvoir politique et l'entreprise. A priori, une telle asymétrie peut être perçue comme dommageable. Mais si l'Etat n'est plus bienveillant et qu'il a des agendas privés, on peut en fait améliorer le bien-être social en créant cette asymétrie d'information qui lui rend plus difficile la poursuite de ses agendas privés. 
C'est ainsi également que j'ai essayé de théoriser la controverse sur la tarification au coût marginal (Laffont, 1998). Hotelling (1939) avait en effet suggéré de tarifer au coût marginal en finançant les déficits par l'impôt, ce qui devait selon lui révolutionner les télécoms, l'électricité, etc. Coase quant à lui a soutenu qu'il fallait tarifer au coût moyen. J'ai tenté de revenir aux sources de cette controverse en cherchant d'abord à savoir à qui revenait cette idée de tarification au coût marginal . Selon Hotelling, elle revenait à Dupuit. Or ce dernier ne semble pas soutenir cette idée. Il donne certes tous les instruments intellectuels pour comprendre qu'un optimum de Pareto doit correspondre à la tarification au coût marginal mais, quand il s'agit de faire de la politique économique, il montre qu'il faut en fait tarifer au coût moyen. Cette idée est aussi présente chez Walras et Edgeworth, tous deux faisant référence à Smith. Après quelques recherches, j'ai trouvé chez Smith des pages peu connues qui expliquent pourquoi, pour des raisons d'économie politique, il faut tarifer au coût moyen. Il a un paragraphe dans lequel il montre que si on tarifie au coût marginal, les intendants de province vont construire des routes pour desservir leurs propres châteaux (le coût marginal étant nul et l'ensemble financé par les contribuables). C'est ainsi, pourrait-on ajouter, que l'on verra des bâtiments somptueux construits par les conseils généraux dans tous les départements de France.

Ceci nous conduit à proposer des règles d'économie publique qui sont très différentes des règles normatives traditionnelles des économistes. Elles cherchent en effet à résoudre un problème supplémentaire qui est celui de la délégation des décisions. Il se peut donc qu'il y ait des règles magnifiques comme la règle de tarification au coût marginal mais qui sont très manipulables par les hommes politiques auquel cas on préférera, en tant qu'économiste, faire des propositions qui s'écartent de celles d'un optimum traditionnel. C'est ainsi qu'on pourra vouloir renoncer à une tarification Ramsey-Boiteux et préférer une tarification avec surcharge uniforme sur les prix si cela peut diminuer les lobbies et la manipulation politique.

Pour conclure, l'avenir immédiat de l'économie publique théorique me semble pouvoir emprunter trois voies :

Premièrement, et c'est ce que je conseille pour commencer, il serait utile d'appliquer de façon systématique l'approche de la théorie des mécanismes à tous les domaines de l'économie dans lesquels cet exercice n'a pas été fait : économie publique, économie de la santé, économie de l'éducation, économie de l'environnement. Cet exercice est très utile car il permet en particulier de bien identifier les sources des problèmes informationnels de l'intervention publique. Quand bien même la démarche n'est pas forcément réaliste, elle fournit un cadre de référence normatif dans un monde d'information incomplète, cadre que l'on a la chance d'avoir et qu'il serait dommage d'ignorer. (Continuer de comparer les politiques avec ce qu'on ferait dans un monde à information parfaite ne présente en effet plus aucun intérêt). Dans cette démarche, on compare ainsi les politiques publiques au pire avec ce que ferait un régulateur qui serait soumis aux mêmes contraintes informationnelles quand bien même on doit continuer de supposer qu'il est parfaitement rationnel. On a, en tout état de cause, fait un pas de plus vers le réalisme et peut-être se fera-t-on moins d'illusions sur ce que peut vraiment réaliser l'État.

Alors, une fois que cette étape est franchie, si on veut être plus concret, il faut relâcher les hypothèses de cette approche et ce, pas à pas. Que se passe-t-il dans le modèle si l'État ne peut plus s'engager à long terme ? Ou bien que l'on a des coûts de communication? Ou encore si l'on a des comportements de groupes ou d'autres formes de limites contractuelles comme la non-vérifiabilité. Et pourquoi je dis pas à pas ? Parce que prendre deux de ces hypothèses à la fois c'est courir le risque d'être incohérent, puisque nous ne savons pas tracer l'application claire qui va de la rationalité limitée, entre autres, vers ces raccourcis que sont ces contraintes. Si l'on en prend plus d'une à la fois, elles risquent de n'être plus compatibles avec les contraintes fondamentales. De l'importance donc du pas à pas.

Enfin, troisième étape, il faut mieux modéliser le contrôle démocratique des hommes politiques pour construire cette économie politique de l'économie publique qui, peut-être, amènera les économistes à faire des propositions qui seront un peu plus prises au sérieux par la société.

En conclusion, en étant plus réaliste sur les limites de l'intervention de l'État soulignées précédemment, on pourra alors construire une vraie défense de l'économie publique qui échappe à la fois aux mirages des uns et au dogmatisme des autres. 


\section{Bibliographie}

Aghion P. et Bolton P. (1992). "An Incomplete Contracts Approach to Financial Contracting", Review of Economic Studies, 59, pp. 473-493.

Aspremont C. d' et Gérard-Varet L.A. (1979). "Incentives and Incomplete Information", Journal of Public Economics, 11 , pp. 25-45.

Baron D. et Myerson R . (1982). "Regulating a Monopolist with Unknown Costs", Econometrica, 50, pp. 911-930.

Crémer J. et McLean R. (1988). "Full Extraction of the Surplus in Bayesian and Dominant Strategy Auctions", Econometrica, 56, pp. 1247-1258.

Dasgupta P., Hammond P. et Maskin E. (1980). “A Note on the Imperfect Information and Optimal Pollution Control", Review of Economic Studies, 47, pp. 857-860.

Dewatripont M. (1989). "Renegotiation and Information Revelation Over Time", Quarterly Journal of Economics, 103, pp. 589-620.

Gasmi F., Ivaldi M. et Laffont J.J.(1994). "Rent Extraction and Incentives for Efficiency in Recent Regulatory Proposals", Journal of Regulatory Economics, 6, pp. 151-176.

Green J. et Laffont J.J. (1979). Incentives in Public Decision Making, Amsterdam, North Holland.

Grossman S. et Hart O. (1986). "The Costs and Benefits of Ownership: A Theory of Lateral and Vertical Integration", Journal of Political Economy, 94, pp. 691-719.

Groves T. (1973). "Incentives in Teams", Econometrica, 41, pp. 587-601.

Hotelling H. (1939). "The Relation of Prices to Marginal Costs in an Optimum System", Econometrica, 7, pp. 151-155.

Laffont J.J. (1994). "Regulation of Pollution with Asymmetric Information", in T. Graham-Tomasi and Serguson etc.

Laffont J.J. (1998). "Frisch, Hotelling and the Marginal Cost Pricing Controversy", Econometrics and Economic Theory in the $20^{\text {th }}$ Century, The Ragnar Frisch Centennial Symposium, ed. Steinar Ström, Cambridge University Press, pp. 319-342.

Laffont J.J. et Martimort D. (1998). "Collusion and Delegation", Rand Journal of Economics, 29, pp. 208-305.

Laffont J.J.et Maskin E. (1979). “A differentiable approach to expected utility maximizing mechanisms", paru dans "Aggregation and Revelation of Preferences", North Holland.

Laffont J.J. et Rochet J.C. (1998). "Regulation of a Risk Averse Firm", Games and Economic Behavior, 25, pp. 149-173.

Laffont J. J. et Tirole J. (1986). "Using Cost to Regulate Firms", Journal of Political Economy, 95, pp. 921-937.

Laffont J. J. et Tirole J. (1987). "Comparative Statics of the Optimal Dynamic Incentive Contract", European Economic Review, 31, pp. 901-926.

Laffont J. J. et Tirole J. (1993). A Theory of Incentives in Procurement and Regulation, MIT Press.

Laffont J. J. et Tirole J. (1996). "Pollution Permits and Environmental Innovation", Journal of Public Economics, 62, pp. 127-140.

Loeb M. et Magat W. (1979. "A Decentralized Method of Utility Regulation", Journal of Law and Economics, 22, pp. 399-404.
Mailath G. et Postlewaite A. (1990). "Asymmetric Information Bargaining Problems with Many Agents", Review of Economic Studies, 57, pp. 351-368.

McAfee P. et McMillan J. (1987). Incentives in Government Contracting, Toronto, University of Toronto Press.

Moulin H. (1980). "On Strategy Proofness and Single Peakedness", Public Choice, 35, pp. 437-455.

Myerson R. (1981). "Optimal Auction Design", Mathematics of Operations Research, 6, pp. 58-73.

Rob R. (1989). "Pollution Claim Settlements with Private Information", Journal of Economic Theory, 47, pp. 307-333.

Shapiro C. et Willig R. (1990). "Economic Rationales for the Scope of Privatization", DP 41, Princeton University.

Tirole J. (1986). "Hierarchies and Bureaucracies", Journal of Law, Economics and Organization, 2, pp. 181-214.

Wilson R. (1993). Nonlinear Pricing, Oxford University Press. 